\title{
LUCAS Copernicus 2018: Earth-observation-relevant in situ data on land cover and use throughout the European Union
}

\author{
Raphä̈l d'Andrimont ${ }^{1}$, Astrid Verhegghen ${ }^{1}$, Michele Meroni ${ }^{1}$, Guido Lemoine ${ }^{1}$, Peter Strobl ${ }^{1}$, \\ Beatrice Eiselt ${ }^{2}$, Momchil Yordanov ${ }^{1}$, Laura Martinez-Sanchez ${ }^{1}$, and Marijn van der Velde ${ }^{1}$ \\ ${ }^{1}$ European Commission Joint Research Centre (JRC), Ispra, Italy \\ ${ }^{2}$ European Commission, Eurostat (ESTAT), Luxembourg, Luxembourg \\ Correspondence: Raphaël d'Andrimont (raphael.dandrimont@ec.europa.eu) and Marijn van der Velde \\ (marijn.van-der-velde@ec.europa.eu)
}

Received: 3 July 2020 - Discussion started: 12 August 2020

Revised: 25 January 2021 - Accepted: 7 February 2021 - Published: 19 March 2021

\begin{abstract}
The Land Use/Cover Area frame Survey (LUCAS) is an evenly spaced in situ land cover and land use ground survey exercise that extends over the whole of the European Union. LUCAS was carried out in 2006, 2009, 2012, 2015, and 2018. A new LUCAS module specifically tailored to Earth observation (EO) was introduced in 2018: the LUCAS Copernicus module. The module surveys the land cover extent up to $51 \mathrm{~m}$ in four cardinal directions around a point of observation, offering in situ data compatible with the spatial resolution of high-resolution sensors. However, the use of the Copernicus module being marginal, the goal of the paper is to facilitate its uptake by the EO community. First, the paper summarizes the LUCAS Copernicus protocol to collect homogeneous land cover on a surface area of up to $0.52 \mathrm{ha}$. Secondly, it proposes a methodology to create a ready-to-use dataset for Earth observation land cover and land use applications with high-resolution satellite imagery. As a result, a total of 63364 LUCAS points distributed over 26 level-2 land cover classes were surveyed on the ground. Using homogeneous extent information in the four cardinal directions, a polygon was delineated for each of these points. Through geospatial analysis and by semantically linking the LUCAS core and Copernicus module land cover observations, 58426 polygons are provided with level-3 land cover (66 specific classes including crop type) and land use (38 classes) information as inherited from the LUCAS core observation. The open-access dataset supplied with this paper (https://doi.org/10.6084/m9.figshare.12382667.v4 d'Andrimont, 2020) provides a unique opportunity to train and validate decametric sensor-based products such as those obtained from the Copernicus Sentinel-1 and Sentinel-2 satellites. A follow-up of the LUCAS Copernicus module is already planned for 2022. In 2022, a simplified version of the LUCAS Copernicus module will be carried out on 150000 LUCAS points for which in situ surveying is planned. This guarantees a continuity in the effort to find synergies between statistical in situ surveying and the need to collect in situ data relevant for Earth observation in the European Union.
\end{abstract}




\section{Introduction}

The Land Use/Cover Area frame Survey (LUCAS) is an evenly spaced in situ land cover and land use data collection exercise that extends over the whole of the European Union (EU) (Gallego and Delincé, 2010; Eurostat, 2018c). LUCAS has been carried out in 2006, 2009, 2012, 2015, and 2018. During these five campaigns, a total of 1351293 points at 651780 unique locations were surveyed and 5.4 million photos were collected. On each of these surveyed points, observations were recorded on up to 109 variables. The combination of the information collected in the five LUCAS surveys has resulted in the most comprehensive in situ database on land cover and land use in the EU (d'Andrimont et al., 2020).

LUCAS in situ data collection was designed for EU-wide standardized reporting of land cover and land use area statistics and not for training and validation of remote sensing data algorithms. The LUCAS activity is complementary to the CORINE Land Cover (CLC) inventory that collects land cover data by interpreting satellite images and orthophotos. In addition, in 2018 the Copernicus High Resolution Layers (HRL) were produced to provide information about different land cover characteristics. Five HRLs describe some of the main land cover characteristics: impervious (sealed) surfaces (e.g., roads and built-up areas), forest areas, grasslands, water and wetlands, and small woody features.

In the scientific community, LUCAS has been widely used for soil studies thanks to the topsoil survey module (Orgiazzi et al., 2018). LUCAS data have also already been valuable in the context of land cover and land use research and remote sensing specifically. Esch et al. (2014) used the data for crop-type mapping in the north of Germany. Zillmann et al. (2014) provided an accuracy assessment of grassland mapping in Hungary based on LUCAS. Mack et al. (2017) used Landsat time series along with LUCAS in situ data to generate a land use and land cover product for Germany. Leinenkugel et al. (2019) assessed the potential of open geodata including LUCAS to generate land use and land cover products from multi-temporal Landsat satellite observations over three European sites. Pflugmacher et al. (2019) recently demonstrated the potential of using LUCAS to map panEuropean land cover (13 classes) with Landsat data. Close et al. (2018) provided a Sentinel-2 LUCAS-based classification over southern Belgium in the context of Land Use, Land-Use Change, and Forestry (LULUCF) monitoring. According to Weigand et al. (2020), LUCAS in situ data are a suitable source for classifying high-resolution Sentinel-2 imagery at a large scale. Weigand et al. (2020) tested the accuracy of different pre-processing approaches of the LUCAS data based on positioning and semantic selection. These studies highlight that there is an interest and value to the remote sensing research community in using LUCAS in situ data. Nevertheless, the LUCAS core protocol has major limitations in terms of spatial scale and representativeness when it comes to collecting in situ data for calibration, training, and/or validation of EO products.

While LUCAS survey data had been valuable in providing in situ observations relevant for remote sensing as highlighted, the LUCAS survey was designed to collect statistics and thus has inherent shortcomings when used in the context of EO. In 2018, a new "LUCAS module" specifically tailored to Earth observation (EO) was introduced: the "LUCAS Copernicus module" (words in quotation marks are defined in the glossary in Appendix B). The Copernicus module was designed to improve the value of LUCAS in situ surveying for EO for three specific reasons described hereafter.

First, in situ observations recorded with a high precision are needed to ensure the quality of EO applications and the development of services relying on geo-location. Second, the collection of the in situ data has to be done through protocols that are compatible with decametric sensors. Specifically, the spatial extent of the observation needs to be designed according to the spatial resolution of common EO sensors. Third, the diversity of land cover and land use in the EU needs to be represented in the thematic diversity of the information collected in situ. Comprehensive and thematically rich in situ data can lead to better classifiers and more accurate multi-temporal land surface mapping. Finally, the representative, comprehensive, and precisely geo-located in situ data available over larger areas need to be made available with an open-access license.

Free and open accessibility is in fact essential for contributing to the creation of common in situ datasets and protocols as currently pursued by, for example, the Land Product Validation (LPV) of the Working Group on Calibration and Validation of the Committee on Earth Observation Satellites (CEOS) and by the Joint Experiment for Crop Assessment and Monitoring (JECAM). The availability of such datasets acquired with transparent protocols is key to assess the quality of EO products resulting from various public and commercial activities. Thus, the Copernicus module gives the opportunity to further integrate the classical LUCAS survey purpose of collecting statistically representative information with the need to collect in situ data to produce better EOderived products, specifically for the EU's Copernicus program. The Copernicus module equips the EU with an in situ dataset specifically fitting EO land application monitoring, allowing the development of consistent land monitoring at the EU level.

While data from the Copernicus module have a been available since 2019, they have not been used in EO applications (to the best of our knowledge). This study is reducing the complexity of the data to ease the uptake of LUCAS Copernicus data by the remote sensing community.

This paper describes and provides the LUCAS Copernicus data in a ready-to-use format. More specifically, this study (i) describes the LUCAS 2018 Copernicus in situ survey protocol, (ii) presents a methodology to produce polygons from the surveyed data to be used in EO studies, (iii) proposes 
a method to inherit more detailed information from the LUCAS core, and (iv) highlights the added value of the survey in order to derive a simplified protocol for the LUCAS Copernicus module that will be integrated in future LUCAS surveys (e.g., in 2022).

\section{LUCAS 2018 data}

The survey consists of a two-phase sampling. In the first phase, 1.1 million geo-referenced points are systematically drawn forming a $2 \times 2 \mathrm{~km}^{2}$ grid, i.e., one point every $2 \mathrm{~km}$ in the EU. The points are then stratified according to land cover classes to allow the second phase of sampling. In 2018, this resulted in 337854 points for which statistical information is collected by surveyors in the field or by photo interpretation in the office. The sampling design methodology used for the LUCAS 2018 survey is described in detail in Scarnò et al. (2018). The grid is static and includes 1090863 points stratified according to land cover class and is available in CSV format from Eurostat (2019b). For a detailed description of the grid data see Eurostat (2018a), and for technical details about the stratification see Eurostat (2018b).

In 2018, the campaign involved more than 1300 actors including more than 900 surveyors and lasted for 23 months. The actual in situ data collection occurred between March and September 2018. The raw data have been available online since May 2019 (Eurostat, 2019a) as a downloadable CSV table with 97 columns and 337854 records (Table 4 presents the attribute names of the 97 original fields; a record descriptor is available in Eurostat, 2019c; the detailed survey instructions in Eurostat, 2018d). Out of the 337854 points surveyed in $2018,23 \%$ points had been included in three previous surveys $(2009,2012$, and 2015), $25 \%$ had already been surveyed once or twice before (e.g., in 2009 and 2015), and the remaining $52 \%$ of the points were new entries. In the LUCAS 2018 survey, $70.45 \%$ of the points were surveyed in situ, and $29.54 \%$ were obtained through the interpretation of detailed ortho-photos (Tables 1 and A1).

Eurostat has carried out LUCAS surveys every 3 years with the survey design ever evolving; however the LUCAS core component (i.e., the identification of the point, and the surveying of specific variables on different aspects of land cover, land use, and land and water management; Eurostat, 2018d) has remained comparable for all five surveys. At each LUCAS point, standard variables are collected including land cover, land use, environmental parameters, and landscape photos. In addition to the core variables collected, other specific modules were carried out on demand such as (i) the transect of $250 \mathrm{~m}$ to assess transitions of land cover and existing linear features $(2009,2012,2015)$, (ii) the topsoil module (2009, 2012 (partly), 2015, and 2018), (iii) the grassland (2018), and (iv) the Copernicus module (2018).

Out of the 337854 LUCAS points sampled in 2018 (combining in situ and photo-interpreted points, Table A1), the sample of the Copernicus module was a third-phase sampling nested in the two-phase sampling scheme. The Copernicus module was planned for 90620 points and actually executed for 63364 points (Table 1). For 27256 (30.08\%) planned points, the surveyors did not manage to reach the point to make the observation, for example due to natural or humanmade obstacles. Therefore, the Copernicus module was carried out in situ for a total of 63364 points, thus corresponding to $69.92 \%$ of the planned Copernicus points.

\section{LUCAS 2018 Copernicus protocol}

In the LUCAS core protocol, the surveyor aims to get as close as possible to the theoretical point. The surveyor then provides the so-called LUCAS core observations for the "LUCAS theoretical grid" point from the location that the surveyor was actually able to reach. Thus, although typically close to each other, the nominal geolocation of a LUCAS point may not exactly coincide with the actual observation location, which is not recorded for LUCAS core points. As an illustrative example, the observation is made from an unknown location and assigned to the LUCAS nominal point in red in Fig. 1. The exact geolocation of the surveyor observation is recorded only in the corresponding LUCAS Copernicus entry (green point in Fig. 1). The "LUCAS theoretical grid" point observation is representative for a circle of $1.5 \mathrm{~m}$ radius. In some specific cases, the window of observation is extended to a $20 \mathrm{~m}$ radius whenever the land cover at the point is heterogeneous (Eurostat, 2018d). This occurs in areas such as permanent crops (B7X, B8X, except nurseries B83) where the parcels of permanent crops contain trees or other plants along with bare soils and/or grassland or another crop; in woodland (CXX); shrubland (DXX) where a mix of, for example, shrubs and trees might occur; in grassland $(\mathrm{EXX})$ where land features may alternate (e.g., grassland with trees); in bare land (FXX); and in wetland (HXX). Given the mentioned protocol, two main drawbacks of the LUCAS core observations are apparent for their use in the context of EO applications.

The first limitation is that the observation corresponds to a fraction ( $7 \%$ ) of a Sentinel-1 and Sentinel-2 pixel (the circle with $1.5 \mathrm{~m}$ radius, thus representing an area of $7.07 \mathrm{~m}^{2}$ ) and is thus not directly usable with such decametric sensors. Indeed, the $10 \mathrm{~m}$ pixel (i.e., $100 \mathrm{~m}^{2}$ ) could be covered by different land covers while the LUCAS observation only captures one. This jeopardizes the use of LUCAS core observations for training and validation when building EO-derived products. The second limitation refers to GPS geo-location survey inaccuracies that are comparable to the representative area, making the information unsuitable. To address these limitations, the LUCAS Copernicus module collected the exact geolocation of the observation as well as information on the spatial extent and homogeneous continuity of the land cover 
Table 1. LUCAS 2018 points totaling 337854 points. The points were either surveyed in situ ( $238014,70.45 \%)$, photo-interpreted in the office $(99803,29.54 \%$ ), or not surveyed (i.e., "in situ PI not possible", "out of national territory", or "out of EU28”). The Copernicus module was collected for a subset of the in situ points in addition to the LUCAS core protocol collected for any in situ point.

\begin{tabular}{lrrr}
\hline & LUCAS core points & LUCAS core and Copernicus & Total \\
\hline In situ & $147394+27,256^{*}$ & 63364 & 238014 \\
Office photo-interpreted & 99803 & - & 99803 \\
Others & 37 & - & 37 \\
\hline Total & 274490 & 63364 & 337854 \\
\hline
\end{tabular}

* Planned in the Copernicus module, but the Copernicus survey was not possible and thus solely surveyed as a point.

(LC) observed around the point, making it suitable for use in EO applications.

More specifically, the following additional data are collected on the LUCAS Copernicus surveyed points: (i) the measured location of the observation and (ii) the land cover (level-2) extent up to $51 \mathrm{~m}$ from the point in the four cardinal directions (N, W, S, E), as well as the neighboring LC. Note that the surveyor records $51 \mathrm{~m}$ to indicate that the land cover is homogeneous for more than $50 \mathrm{~m}$. However, as the exact extent is not reported, we conservatively set it to the minimum extent of $51 \mathrm{~m}$. Figure 1 illustrates the Copernicus protocol for one point, and the respective collected data are shown in Table 2. On the basis of these LUCAS Copernicus observations, a quadrilateral polygon with homogeneous LC can be constructed. As part of the Copernicus module, the surveyor collects 13 additional variables and three types of observations (Table 2): the level-2 LC (one variable), the extent of the Copernicus land cover (LUCAS LC classification at level 2) registered at the point reached in the field (four variables), the next land cover (up to $50 \mathrm{~m}$ ) (four variables), and the breadth of the next land cover (four variables). The breadth corresponds to the percent of the width of the land cover in this sector, as visible on the landscape photo (i.e., landscape photos taken in each cardinal direction: N, E, S, $\mathrm{W})$. This means that the breadth is $100 \%$ if the next LC is seen all over the photo from one side to the other. If the next land cover is not visible on the photo because it is completely behind a linear feature (e.g., hedge) or because it is completely hidden by the terrain, then the next land cover is to be recorded but the breadth is $0 \%$. For more information about the breadth and the next land cover, see Eurostat (2018d).

The following sections describe how the LUCAS Copernicus data are prepared and cleaned to obtain the ready-touse dataset provided with this paper. The following workflow was done in R (Code and Data availability; see Sect. 6).

\subsection{Adding an explicit LUCAS land cover and land use legend}

The LUCAS land cover classification is hierarchical and contains four levels briefly described hereafter (for a detailed description, see the Technical reference document C3 Classifi-
Table 2. Example of information collected by the Copernicus protocol (for point with ID 45223358). The Copernicus protocol collects observations on 13 variables: land cover (LC) at LUCAS legend level 2 (here B3 is "non-permanent industrial crops"), the extension of the $\mathrm{LC}$ in the four cardinal directions (up to $50 \mathrm{~m}, 51$ means more than $50 \mathrm{~m}$ ), the breadth of the next LC in the four cardinal directions $(\%)$, and the next LC in the four directions (here, E2 means "grassland without tree/shrub cover" in the $\mathrm{N}$ and $\mathrm{W}$ ). Figure 1 shows how this information is used to build the geometries of the Copernicus polygon with homogeneous LC. The radial distance $d$ is measured between the Copernicus point and the next LC, with " 888 " and " 8 " meaning "not relevant".

\begin{tabular}{l|lll}
\hline & Copernicus LC: B3 & \\
\hline & Extension $(d)$ & Breadth of next & Next LC, if $d \leq$ \\
& of Copernicus & LC in percent, & $50 \mathrm{~m}$ \\
& LC in cardinal & if $d \leq 50 \mathrm{~m}$ & \\
& directions in & & \\
& meters & & \\
\hline $\mathrm{N}$ & 30 & 15 & E2 \\
$\mathrm{E}$ & 51 & 888 & 8 \\
$\mathrm{~S}$ & 51 & 888 & 8 \\
$\mathrm{~W}$ & 45 & 50 & E2 \\
\hline
\end{tabular}

cation, Eurostat, 2018e). The land cover classification system is subdivided into eight main level-1 land cover categories: artificial land, cropland, woodland, shrubland, grassland, bare land, water, and wetlands. The level-2 legend contains 26 classes (e.g., 8 under level-1 B cropland) and level-3 comprises 73 classes (e.g., 9 under level-2 B1 cereals). Only a limited number of observations have level-4 land cover information distributed into 205 classes ("LC1_SPEC" field in the data). Similarly, the land use comprises 40 subclasses.

To facilitate the usability of the data, in addition to the code describing the land use or land cover (e.g., B21 or U112), an explicit legend label was added to the dataset provided with this paper. This was done by adding nine explicit label fields (Table 4) to the data for the LC and LU legend.

In the results section, details on the hierarchical legend structure classes are also provided (Table 3 on level-2 legend, Fig. 5 on level-3 legend ("LC1" field in the data), and 


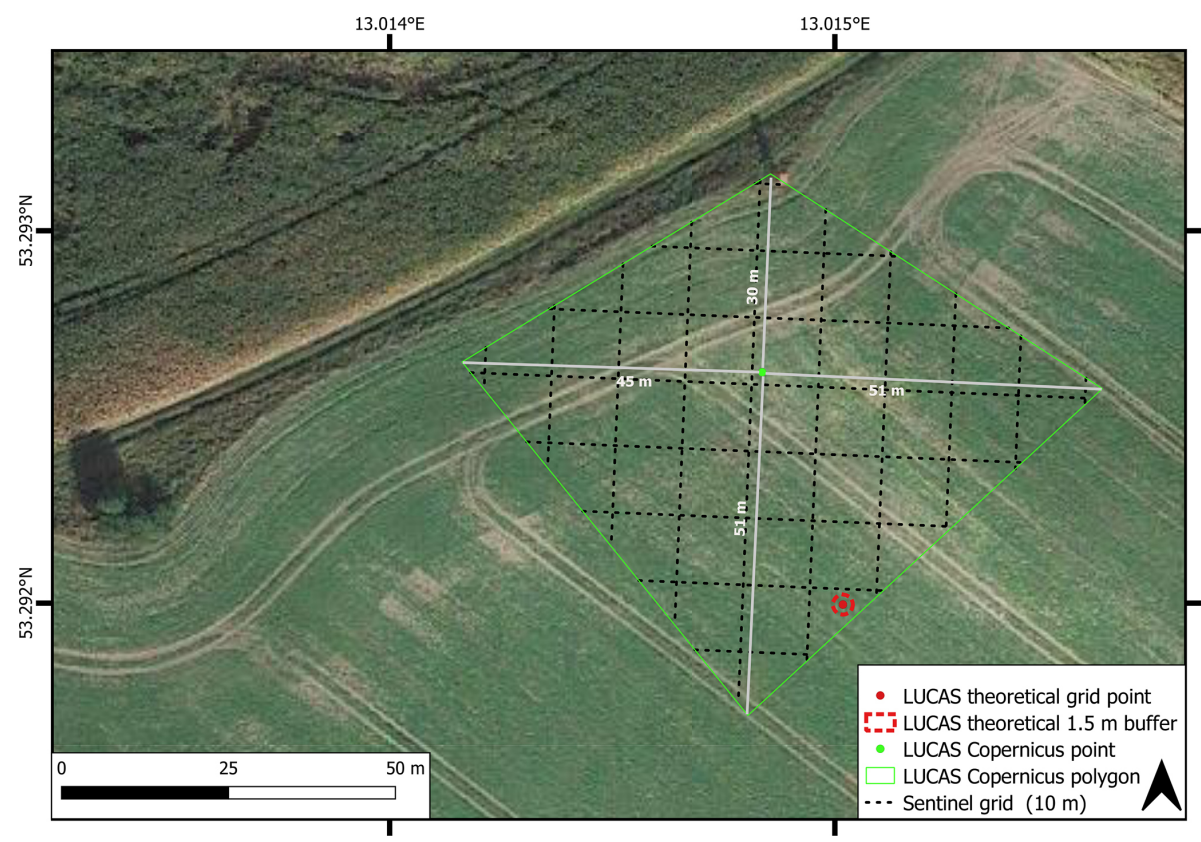

Figure 1. Building the Copernicus polygon geometry (example for point ID 45223358). The collected Copernicus variables (Table 2) are used to build the geometry of the Copernicus polygon. As the LUCAS theoretical point is inside the Copernicus polygon, the LC legend of the LUCAS theoretical observation (here B32 - rape and turnip rape) could be inherited to the Copernicus polygon (B3 - non-permanent industrial crops) as described in Sect. 4. The background RGB imagery is obtained from map data (C2019 Google.

the 40 land use sub classes as shown by the level- 3 distribution of the Copernicus polygons in Table A2), "LU1" in the data).

\subsection{Constructing the LUCAS Copernicus polygon}

On the basis of the LUCAS Copernicus observations, a polygon with homogeneous LC can be constructed. In order to generate the LUCAS Copernicus polygon, the Copernicus point (i.e the effective location of the surveyor, i.e., "GPS_LAT" and "GPS_LONG") is defined as the center to build a quadrilateral for each point. The location of this point is first projected in the Lambert azimuthal equal-area projection coordinate reference system (ETRS89-LAEA). Second, the four distances (N, W, S, E) measured by the surveyor are added to the point in the four respective cardinal directions, resulting in 63364 irregular quadrilaterals (Table 1). The quadrilateral diagonals can measure up to $102 \mathrm{~m}$ but are smaller if the surveyor found a field boundary within $51 \mathrm{~m}$ of the LUCAS Copernicus point.

\subsection{Quality check}

While the Copernicus protocol was implementable for 63364 polygons, several surveyed polygon locations (i.e., LUCAS Copernicus polygon as defined by "GPS_LAT" and "GPS_LONG") were either missing or wrong. The missing locations could be flagged for 67 polygons ("GPS_PREC" = 8888, "GPS_LAT" $=0$, or "GPS_LONG" $=0$ ). In addition to these, 10 polygons were discarded because the surveyor geolocation ("GPS_LAT", "GPS_LONG") was far away from the nominal location ("TH_LAT" , “TH_LONG”), i.e., difference larger than $0.1^{\circ}$ (i.e., about $7.1 \mathrm{~km}$ in the center of the EU). In addition to the missing GPS-measured locations, some macro errors were flagged and removed by selecting polygons for which the longitude and latitude differences between the GPS measured location and theoretical location ("TH_LAT", "TH_LONG") are larger than $0.1^{\circ}$. This allows us to flag and remove 10 polygons which are all wrongly located because of the "GPS_EW" field (i.e., GPS observation east-west). This location quality check permits us to flag and remove a total of 75 polygons resulting in a final total of 63287 polygons.

\subsection{Resulting LUCAS Copernicus data}

The 63287 Copernicus polygons surveyed are published along with this paper. They are distributed among 26 level-2 LC classes (Table 3) in eight level-1 LC classes (see map in Fig. 3).

The homogeneous area of the 63287 polygons ranges between 0.005 and 0.52 with an average of 0.33 ha (Fig. 2) corresponding to $3210 \mathrm{~m}$ pixels. Half of the polygons are larger than $0.33 \mathrm{ha}$. Also, the third quartile corresponds to the maximum area of $0.52 \mathrm{ha}$, which is the maximum area possible for a rhombus with diagonals of $102 \mathrm{~m}(51 \mathrm{~m}+51 \mathrm{~m})$. Among the 63287 , it is worth mentioning that 21657 polygons (i.e., $34.2 \%$ ) have an area greater than 0.5 ha, i.e., thus 
Table 3. Distribution of level-2 land cover classes of the resulting LUCAS Copernicus polygons $(N=63287)$.

\begin{tabular}{|c|c|c|}
\hline Class & Label & No. of LUCAS Copernicus polygons \\
\hline Roofed built-up areas & A1 & 12 \\
\hline Artificial non-built up areas & $\mathrm{A} 2$ & 374 \\
\hline Other artificial areas & $\mathrm{A} 3$ & 21 \\
\hline Cereals & $\mathrm{B} 1$ & 12774 \\
\hline Root crops & $\mathrm{B} 2$ & 877 \\
\hline Non-permanent industrial crops & B3 & 2435 \\
\hline Dry pulses, vegetables, and flowers & B4 & 767 \\
\hline Fodder crops & B5 & 2757 \\
\hline Permanent crops: fruit trees & B7 & 817 \\
\hline Other permanent crops & B8 & 1148 \\
\hline Broadleaved woodland & $\mathrm{C} 1$ & 8481 \\
\hline Coniferous woodland & $\mathrm{C} 2$ & 5996 \\
\hline Mixed woodland & $\mathrm{C} 3$ & 4484 \\
\hline Shrubland with sparse tree cover & D1 & 1308 \\
\hline Shrubland without tree cover & D2 & 1546 \\
\hline Grassland with sparse tree/shrub cover & E1 & 2078 \\
\hline Grassland without tree/shrub cover & E2 & 13053 \\
\hline Spontaneously re-vegetated surfaces & E3 & 2608 \\
\hline Rocks and stones & $\mathrm{F} 1$ & 35 \\
\hline Sand & $\mathrm{F} 2$ & 30 \\
\hline Lichens and moss & F3 & 3 \\
\hline Other bare soil & $\mathrm{F} 4$ & 1503 \\
\hline Inland water bodies & G1 & 1 \\
\hline Inland running water & G2 & 9 \\
\hline Inland wetlands & $\mathrm{H} 1$ & 164 \\
\hline \multirow[t]{2}{*}{ Coastal wetlands } & $\mathrm{H} 2$ & 6 \\
\hline & Total & 63287 \\
\hline
\end{tabular}

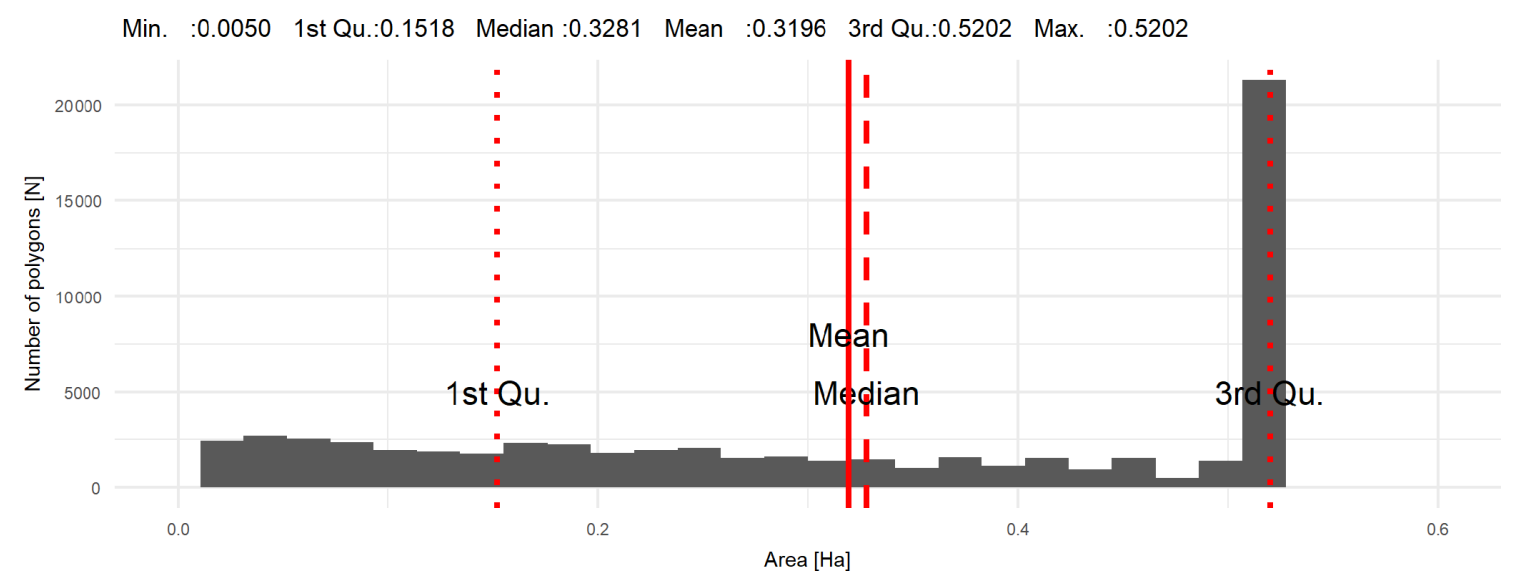

Figure 2. Distribution of the area of the LUCAS Copernicus polygons in hectares $(N=63287)$. On average, the polygon covers an area of 0.33 ha. 


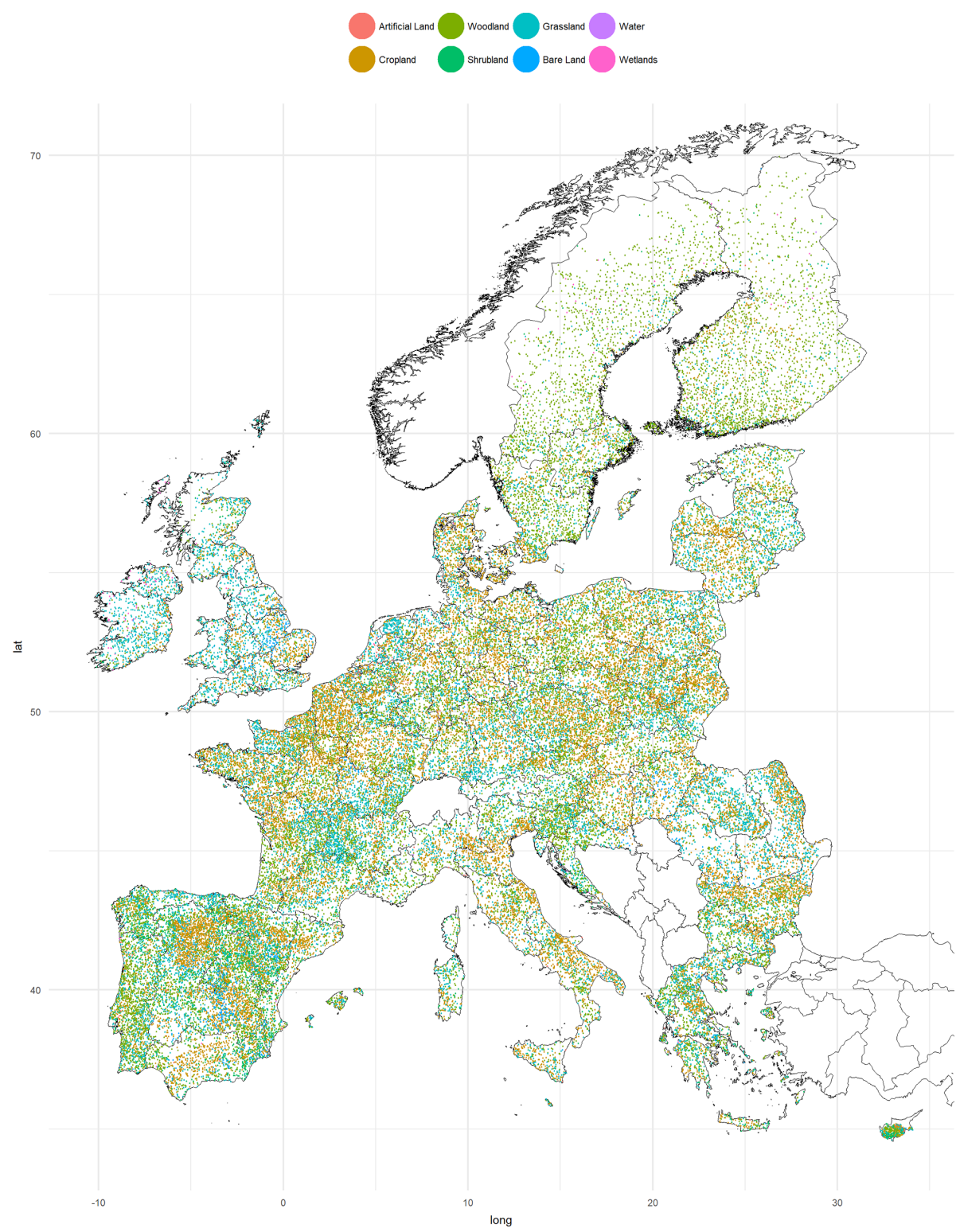

Figure 3. Map of LUCAS Copernicus polygons $(N=63287$ ) surveyed in 2018 per level-1 land cover class over the EU28.

corresponding to almost fifty $10 \mathrm{~m}$ pixels depending on the orientation. These characteristics make the obtained spatial data well suited for training and validation of products based on decametric (i.e., $0.01 \mathrm{ha}$ ) and even subdecametric remote sensing sensors.

\section{Linking LUCAS core data to Copernicus polygons}

A set of rules were defined to link LUCAS core and LUCAS Copernicus data and thus enrich the LUCAS Copernicus set of information. The rationale is that if the LUCAS theoretical point location falls within the LUCAS polygon, the LUCAS-core-surveyed attributes at the theoretical point could be inherited by the LUCAS Copernicus polygon. This condition is satisfied by the vast majority of the polygons (60 134 points, i.e., $95.02 \%$ ). In addition, to filter out suspicious data points where the LUCAS core and Copernicus information were not in agreement despite being spatially consistent, we retained only those points where the reported Copernicus level-2 land cover observed is the same as the one reported for the LUCAS theoretical point (50 417 points, i.e., $95.47 \%$ ). This happens when a surveyor can observe the LUCAS theoretical point from a distance but makes the Copernicus observation at the actual point that was reached. At this Copernicus point, the land cover does not correspond to the land cover of the LUCAS theoretical point. Among the 63287 Copernicus polygons available with this paper, 58426 polygons (i.e., $92.23 \%$ ) fulfill both requirements 


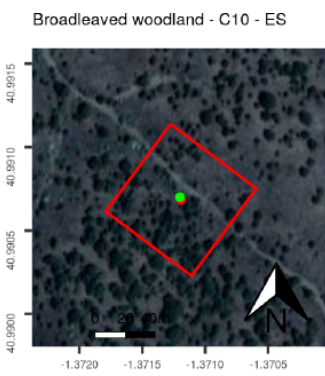

(a)

Non built-up area features - A21 - SE

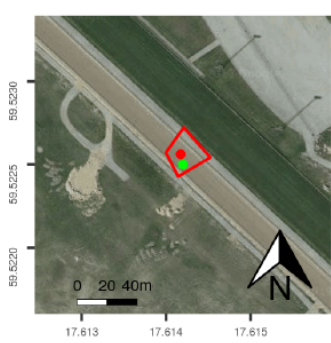

(e)

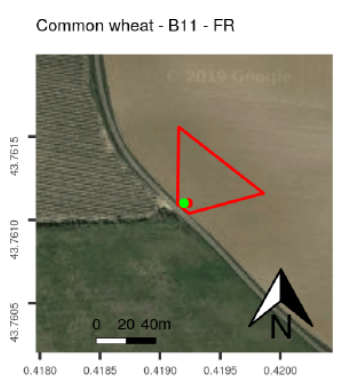

(b)

Grassland without tree/shrub cover - E2O - S

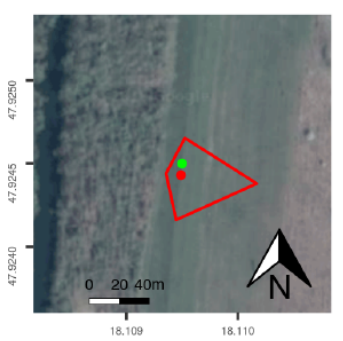

(f)

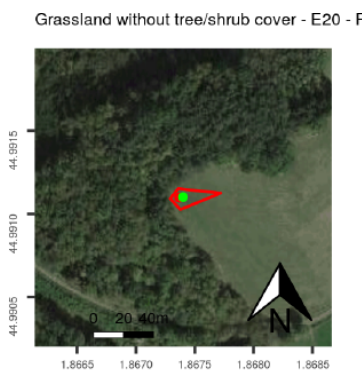

(c)

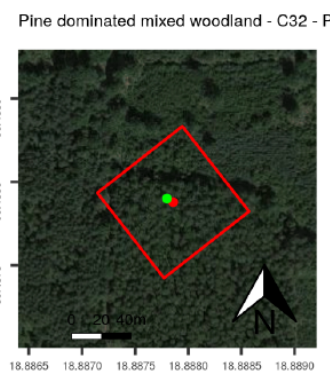

(g)

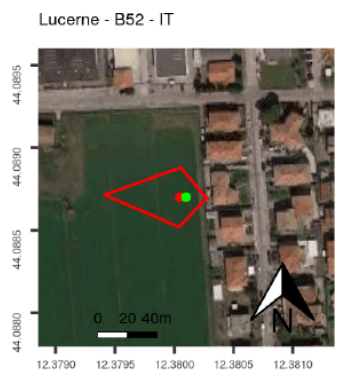

(d)

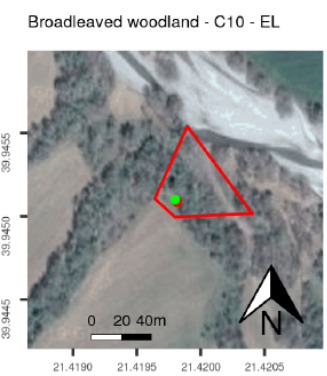

(h)

Figure 4. Examples of LUCAS Copernicus built polygons. The green point is the theoretical LUCAS point. The red point is the GPS location of the Copernicus surveyor. Polygons are built using distances in the N, E, S, and W directions collected on the ground. The background RGB imagery is obtained from map data (C2019 Google.

(condition "CPRN_LC_SAME_LC1" and condition "LUCAS_CORE_INTERSECT" in the provided dataset) and are thus flagged as "COPERNICUS_CLEANED" in the data. For these polygons, the more detailed level-3 land cover class of the LUCAS core can be inherited by the LUCAS Copernicus polygon ("COPERNICUS_CLEANED" is "TRUE"). Figure 4 illustrates the variety in shapes of the constructed quadrilateral Copernicus polygons as projected on top of satellite imagery for different land cover types. The resulting polygons are distributed over 66 specific LC classes as shown in Fig. 5. Similarly, the level-3 land use (LU) is also available distributed in 38 classes organized in four main classes (see Table A2).

\section{Discussion}

The LUCAS Copernicus polygons and data compiled and presented here can provide valuable information for a variety of topics and applications. The LUCAS Copernicus polygons can provide valuable information to extract land-coverspecific surface radiometric and temporal signatures as measured by different sensors, in the multispectral, thermal, and microwave ranges, for different land cover types. This is particularly relevant for land covers exhibiting a dynamic signal (e.g., forests, grasslands, crops) that is modulated by climatic and agroecological conditions, which are well sampled in this EU-wide dataset. As an illustration, the dataset was used by Meroni et al. (2021) to extract crop-specific land surface phenology from Sentinel-1 and Sentinel-2. The dataset can serve various EO-based applications, among many others: train classification algorithms for land cover mapping using existing sensors (e.g., Sentinel 1 and 2, Landsat, QuickBird, ASTER, WorldView), validate land cover products centered on 2018 (e.g., the Copernicus High Resolution Layers), study land-cover-specific land surface processes (e.g., phenology), and develop algorithms to monitor crop and grassland management practices. Future surveys could consider drones to collect high-resolution NADIR view observations concomitant with the survey date. Such data could then be used a posteriori to collect training data for landscape elements difficult to monitor with decametric sensors and could thus provide training data for future research using very high-resolution satellite observations.

With this paper we provide LUCAS Copernicus polygons constructed at 63287 locations. In addition, we provide a dataset that benefits from inheriting attributes collected at those same points via the LUCAS core protocol. This results in 58426 Copernicus polygons, discarding a total of 4861 polygons.

The LUCAS Copernicus module is also planned to be carried out during the LUCAS 2022 survey. However, a simplified protocol has been designed for the LUCAS 2022 survey. In this protocol, the observations on the distance of homogeneous LC from the point and the LC remain, but obser- 
A11 - Buildings with 1 to 3 floors

A13 - Greenhouses

A21 - Non built-up area features

A22 - Non built-up linear features

A30 - Other artificial areas

B11 - Common wheat

B12 - Durum wheat

B13 - Barley

B14 - Rye

B15 - Oats

B16 - Maize

B17 - Rice

B18 - Triticale

B19 - Other cereals

B21 - Potatoes

B22 - Sugar beet

B23 - Other root crops

B31 - Sunflowe

B32 - Rape and turnip rape

B33 - Soya

B34 - Cotton

B35 - Other fiber and oleaginous crops

B36 - Tobacco

B37 - Other non-permanent industrial crops

B41 - Dry pulses

B42 - Tomatoes

B43 - Other fresh vegetables

B44 - Floriculture and ornamental plants

B45 - Strawberries

B51 - Clovers

B52 - Lucerne

\& B53 - Other leguminous and mixtures for fodder

B54 - Mixed cereals for fodde

B55 - Temporary grasslands

B71 - Apple fruit

B72 - Pear fruit

B73 - Cherry fruit

B74 - Nuts trees

B75 - Other fruit trees and berries

B76 - Oranges

B77 - Other citrus fruit

B81 - Olive groves

B82 - Vineyards

B83 - Nurserie

B84 - Permanent industrial crops

C10 - Broadleaved woodland

C21 - Spruce dominated coniferous woodland

C22 - Pine dominated coniferous woodland

C23 - Other coniferous woodlan

C31 - Spruce dominated mixed woodland

C32 - Pine dominated mixed woodland C33 - Other mixed woodlan

D10 - Shrubland with sparse tree cove

D20 - Shrubland without tree cove

E10 - Grassland with sparse tree/shrub cove

E20 - Grassland without tree/shrub cove

E30 - Spontaneously vegetated surfaces

F10 - Rocks and stones

F20 - Sand

F30 - Lichens and moss F40 - Other bare soil

G21 - Inland fresh running wate

H11 - Inland marshes

H12 - Peatbogs

H21 - Salt marshes

H23 - Intertidal flats

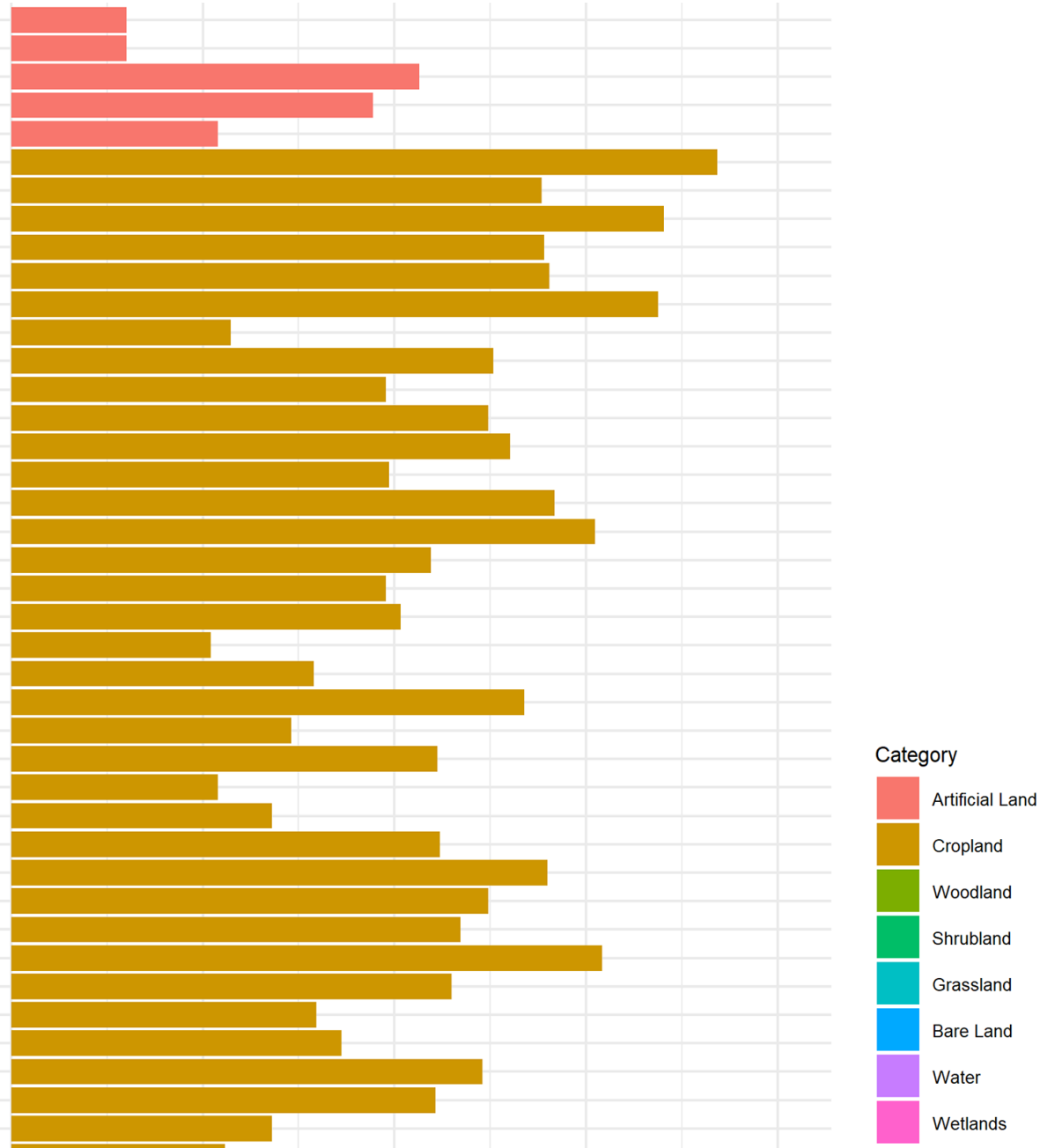

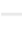


Table 4. The LUCAS Copernicus 2018 dataset is provided as a polygon shapefile along with a table with 120 attributes to be joined based on POINT_ID. Among the 120 attributes, 117 attributes are the original as described in d'Andrimont et al. (2020), and three attributes are obtained as described in Sect. 6.

\begin{tabular}{|c|c|c|}
\hline Origin of attributes & No. & Attribute names \\
\hline Original fields & 117 & $\begin{array}{l}\text { POINT_ID, ID, YEAR, NUTS0, NUTS1, NUTS2, NUTS3, TH_LAT, TH_LONG, OFFICE_PI, } \\
\text { EX_ANTE, SURVEY_DATE, CAR_LATITUDE, CAR_EW, CAR_LONGITUDE, } \\
\text { GPS_PROJ, GPS_PREC, GPS_ALTITUDE, GPS_LAT, GPS_EW, GPS_LONG, OBS_DIST, } \\
\text { OBS_DIRECT, OBS_TYPE, OBS_RADIUS, LETTER_GROUP, LC1, LC1_LABEL, } \\
\text { LC1_SPEC, } 1 \text { _C1_SPEC_LABEL, LC1_PERC, LC2, LC2_LABEL, LC2_SPEC, } \\
\text { LC2_SPEC_LABEL, LC2_PERC, LU1, LU1_LABEL, LU1_TYPE, LU1_TYPE_LABEL, } \\
\text { LU1_PERC, LU2, LU2_LABEL, LU2_TYPE, LU2_TYPE_LABEL, LU2_PERC, PAR- } \\
\text { CEL_AREA_HA, TREE_HEIGHT_MATURITY, TREE_HEIGHT_SURVEY, FEATURE_WIDTH, } \\
\text { LM_STONE_WALLS, CROP_RESIDUES, LM_GRASS_MARGINS, GRAZING, SPE- } \\
\text { CIAL_STATUS, CC_LU_SPECIAL_REMARK, CPRN_CANDO, CPRN_LC, CPRN_LC_LABEL, } \\
\text { CPRN_LC1N, CPRNC_LC1E, CPRNC_LC1S, CPRNC_LC1W, CPRN_LC1N_BRDTH, } \\
\text { CPRN_LC1E_BRDTH, CPRN_LC1S_BRDTH, CPRN_LC1W_BRDTH, CPRN_LC1N_NEXT, } \\
\text { CPRN_LC1S_NEXT, CPRN_LC1E_NEXT, CPRN_LC1W_NEXT, CPRN_URBAN, } \\
\text { CPRN_IMPERVIOUS_PERC, INSPIRE_PLCC1, INSPIRE_PLCC2, INSPIRE_PLCC3, } \\
\text { INSPIRE_PLCC4, INSPIRE_PLCC5, INSPIRE_PLCC6, INSPIRE_PLCC7, INSPIRE_PLCC8, } \\
\text { EUNIS_COMPLEX, GRASSLAND_SAMPLE, GRASS_CANDO, WM, WM_SOURCE, } \\
\text { WM_TYPE, WM_DELIVERY, EROSION_CANDO, SOIL_STONES_PERC, BIO_SAMPLE, } \\
\text { SOIL_BIO_TAKEN, BULK0_10_SAMPLE, SOIL_BLK_0_10_TAKEN, BULK10_20_SAMPLE, } \\
\text { SOIL_BLK_10_20_TAKEN, BULK20_30_SAMPLE, SOIL_BLK_20_30_TAKEN, } \\
\text { STANDARD_SAMPLE, SOIL_STD_TAKEN, ORGANIC_SAMPLE, } \\
\text { SOIL_ORG_DEPTH_CANDO, SOIL_TAKEN, SOIL_CROP, PHOTO_POINT, PHOTO_NORTH, } \\
\text { PHOTO_SOUTH, PHOTO_EAST, PHOTO_WEST, TRANSECT, REVISIT, TH_GPS_DIST, } \\
\text { FILE_PATH_GISCO_NORTH, FILE_PATH_GISCO_SOUTH, FILE_PATH_GISCO_EAST, } \\
\text { FILE_PATH_GISCO_WEST, FILE_PATH_GISCO_POINT }\end{array}$ \\
\hline EO application fields & 3 & CPRN_LC_SAME_LC1,LUCAS_CORE_INTERSECT, COPERNICUS_CLEANED \\
\hline
\end{tabular}

vations on the neighboring LC and breadth of the neighboring LC have been discarded. Despite this simplification, the coverage of the 2022 LUCAS Copernicus module will be expanded to 150000 LUCAS points for which in situ surveying is planned.

\section{Code and data availability}

The data repository https://doi.org/10.6084/m9.figshare. 12382667.v4 (d'Andrimont, 2020) contains the following files:

- LUCAS_2018_Copernicus.zip, compressed files containing the dataset;

-LUCAS_2018_Copernicus_polygons.shp, shapefile of polygons with the "POINT_ID" attribute;

- LUCAS_2018_Copernicus_attributes.csv, CSV file containing the 120 variables including the "POINT_ID";

- ESSD_create_LUCAS_polygons.Rmd, $\mathrm{R}$ markdown script used to generate the data;
- ESSD_manuscript_Tables_and_Figures.Rmd, R markdown script used to generate the figures and tables of the paper;

- LUCAS_2018_Copernicus_ReadMe.txt, short description of the data repository.

The LUCAS Copernicus 2018 dataset is provided as a polygon shapefile along with a CSV table containing 120 attributes. Among the 120 attributes (list in Table 4), 117 attributes are the original fields as described in Eurostat $(2019 \mathrm{c})$, three attributes are obtained as described in Sect. 3.1, and three attributes are obtained as described in the previous Sect. 4 .

To use the data, the attribute "POINT_ID" should be used to join the attribute table of the shapefile and the CSV table. While the Copernicus-related level-2 LC could be used for every polygon, the level-3 LC and LU, along with other LUCAS core information, should be used only for polygons with "COPERNICUS_CLEANED" as "TRUE" as described in the previous section. 


\section{Conclusions}

For the first time, the LUCAS 2018 survey contained a module that was specifically tailored to the needs of EO. The LUCAS Copernicus module collected homogeneous land cover data over areas with a size relevant to $10 \mathrm{~m}$ satellite sensors. A total of 63364 Copernicus polygons were obtained across the EU representing 66 land cover type classes at LUCAS level-2 legend. A follow-up of the LUCAS Copernicus module is planned for 2022. In 2022, a simplified version of the LUCAS Copernicus module is planned to be carried out at 150000 LUCAS points. This guarantees a continuity in the effort to find synergies between statistical in situ surveying and the need to collect in situ data relevant for Earth observation in the European Union. 


\section{Appendix A}

Table A1. Copernicus survey with relation to observation type (OBS_TYPE), observation direction (OBS_DIRECT), and parcel area (PARCEL_AREA_HA).

\begin{tabular}{|c|c|c|c|c|c|}
\hline & & $\begin{array}{r}\text { CPRN_CANDO } \\
\text { Yes }\end{array}$ & No & Not relevant & Total \\
\hline \multicolumn{6}{|c|}{ OBS_TYPE } \\
\hline 1 & In situ $<100 \mathrm{mt}$ & 63364 & 18924 & 121673 & 203961 \\
\hline 2 & In situ $>100 \mathrm{mt}$ & 0 & 2488 & 8671 & 11159 \\
\hline 3 & In situ PI & 0 & 5844 & 17050 & 22894 \\
\hline 4 & In situ PI not possible & 0 & 2 & 23 & 25 \\
\hline 5 & Out of national territory & 0 & 0 & 10 & 10 \\
\hline 6 & Out of EU28 & 0 & 0 & 2 & 2 \\
\hline 7 & In-office PI & 0 & 0 & 99803 & 99803 \\
\hline \multicolumn{6}{|c|}{ OBS_DIRECT } \\
\hline 1 & On the point & 63057 & 23641 & 237089 & 323787 \\
\hline 2 & Look to the north & 251 & 2834 & 8191 & 11276 \\
\hline 3 & Look to the east & 56 & 781 & 1917 & 2754 \\
\hline 8 & Not relevant & 0 & 2 & 35 & 37 \\
\hline \multicolumn{6}{|c|}{ PARCEL_AREA_HA } \\
\hline 1 & Area $<0.1$ & 855 & 3526 & 13965 & 18346 \\
\hline 2 & $0.1 \leq$ area $<0.5$ & 2743 & 2876 & 12979 & 18598 \\
\hline 3 & $0.5 \leq$ area $<1$ & 4168 & 2446 & 12917 & 19531 \\
\hline 4 & $1 \leq$ area $<10$ & 25733 & 8434 & 70371 & 104538 \\
\hline 5 & Area $\geq 10$ & 29865 & 9975 & 136974 & 176814 \\
\hline \multirow[t]{2}{*}{8} & Not relevant & 0 & 1 & 26 & 27 \\
\hline & Total & 63364 & 27258 & 247232 & 337854 \\
\hline
\end{tabular}


Table A2. LUCAS Copernicus level-3 land use distribution.

\begin{tabular}{lr}
\hline Class & No. of LUCAS Copernicus polygons \\
\hline U111 - Agriculture (excluding fallow land and kitchen gardens) & 33603 \\
U112 - Fallow land & 1779 \\
U113 - Kitchen garden & 57 \\
U120 - Forestry & 15990 \\
U130 - Aquaculture and fishing & 1 \\
U140 - Mining and quarrying & 44 \\
U150 - Other primary production & 19 \\
\hline U210 - Energy production & 13 \\
U221 - Manufacturing of food, beverages, and tobacco products & 1 \\
U223 - Coal, oil, and metal processing & 2 \\
U224 - Production of non-metal mineral goods & 3 \\
U225 - Chemical and allied industries and manufacturing & 1 \\
U226 - Machinery and equipment & 1 \\
U227 - Wood-based products & 4 \\
\hline U311 - Railway transport & 12 \\
U312 - Road transport & 163 \\
U313 - Water transport & 3 \\
U314 - Air transport & 12 \\
U315 - Transport via pipelines & 1 \\
U316 - Telecommunication & 2 \\
U317 - Logistics and storage & 17 \\
U318 - Protection infrastructures & 16 \\
U319 - Electricity, gas, and thermal power distribution & 20 \\
U321 - Water supply and treatment & 8 \\
U322 - Waste treatment & 10 \\
U330 - Construction & 31 \\
U341 - Commerce & 38 \\
U342 - Financial, professional, and information services & 2 \\
U350 - Community services & 99 \\
U361 - Amenities, museums, leisure & 326 \\
U362 - Sport & 5086 \\
U370 - Residential & 167 \\
\hline U411 - Abandoned industrial areas & 377 \\
U412 - Abandoned commercial areas & 23 \\
U413 - Abandoned transport areas & 226 \\
U414 - Abandoned residential areas & 4 \\
U415 - Other abandoned areas & \\
U420 - Semi-natural and natural areas not in use & \\
\hline Total & \\
\hline &
\end{tabular}


Appendix B: Glossary

LUCAS theoretical grid

LUCAS core

LUCAS Copernicus module

LUCAS module
The LUCAS theoretical grid is a standard regular $2 \mathrm{~km}$ grid which comprises around 1 million points all over the EU. The LUCAS surveyed points are sampled from this grid.

The LUCAS core variables are the ones collected for each point surveyed. In addition to the core variables, some specific modules could be collected (such as transect, topsoil, grassland, or Copernicus), providing additional specific information.

The Copernicus module is a specific LUCAS module initiated in 2018 to collect the homogeneous and continuous extent of land cover meaningful for EO.

In addition to the LUCAS core variables collected, other specific LUCAS protocols called "modules" were carried out on demand such as (i) the transect of $250 \mathrm{~m}$ to assess transitions of land cover and existing linear features $(2009,2012,2015)$, (ii) the topsoil module (2009, 2012 (partly), 2015, and 2018), (iii) the grassland module (2018), and (iv) the Copernicus module collecting the homogeneous and continuous extent of land cover in a $50 \mathrm{~m}$ radius (2018). 
Author contributions. All the authors processed and analyzed the data, wrote the paper, and provided comments and suggestions on the paper. BE and PS designed the survey methodology. BE and ESTAT are responsible for the LUCAS data collection.

Competing interests. The authors declare that they have no conflict of interest.

Review statement. This paper was edited by Birgit Heim and reviewed by Radoux Julien and Ulf Mallast.

\section{References}

Close, O., Benjamin, B., Petit, S., Fripiat, X., and Hallot, E.: Use of Sentinel-2 and LUCAS Database for the Inventory of Land Use, Land Use Change, and Forestry in Wallonia, Belgium, Land, 7, $154,2018$.

d'Andrimont, R.: LUCAS $2018 \quad$ Copernicus, https://doi.org/10.6084/m9.figshare.12382667.v4, 2020.

d'Andrimont, R., Yordanov, M., Martinez-Sanchez, L., Eiselt, B., Palmieri, A., Dominici, P., Gallego, J., Reuter, H. I., Joebges, C., Lemoine, G., and van der Velde, M.: Harmonised LUCAS in-situ land cover and use database for field surveys from 2006 to 2018 in the European Union, Sci. Data, 7, 352, https://doi.org/10.1038/s41597-020-00675-z, 2020.

Esch, T., Metz, A., Marconcini, M., and Keil, M.: Combined use of multi-seasonal high and medium resolution satellite imagery for parcel-related mapping of cropland and grassland, Int. J. Appl. Earth Obs. Geoinf., 28, 230-237, 2014.

Eurostat: LUCAS Grid Record Descriptor, https: //ec.europa.eu/eurostat/documents/205002/7329820/

LUCAS-Grid-Record-Descriptor.pdf (last access: 22 May 2019), 2018a.

Eurostat: Technical reference document S1: Stratification Guidelines, https://ec.europa.eu/eurostat/documents/205002/7329820/ LUCAS2018_S1-StratificationGuidelines_20160523.pdf (last access: 22 May 2019), 2018b.

Eurostat: LUCAS web site, https://ec.europa.eu/eurostat/web/lucas (last access: 30 August 2018), 2018c.

Eurostat: Technical reference document C-1: Instructions for surveyors, https://ec.europa.eu/eurostat/documents/205002/ 8072634/LUCAS2018-C1-Instructions.pdf (last access: 30 July 2019), 2018d.

Eurostat: Technical reference document C-3: Classification, https://ec.europa.eu/eurostat/documents/205002/8072634/

LUCAS2018-C3-Classification.pdf (last access: 30 July 2019), 2018e.
Eurostat: LUCAS micro data 2018, https://ec.europa.eu/eurostat/ web/lucas/data/primary-data/2018 (last access: 24 May 2019), 2019a.

Eurostat: LUCAS Grid - Eurostat, https://ec.europa.eu/eurostat/ web/lucas/data/lucas-grid (last access: 22 May 2019), 2019b.

Eurostat: LUCAS SURVEY2018 WEB CSV Record Descriptor, https://ec.europa.eu/eurostat/documents/205002/8072634/ LUCAS2018-RecordDescriptor-190611.pdf (last access: 6 August 2020), 2019c

Gallego, J. and Delincé, J.: The European land use and cover area-frame statistical survey, in: Agricultural survey methods, Wiley Online Library, 149-168, https://doi.org/10.1002/9780470665480.ch10, 2010.

Leinenkugel, P., Deck, R., Huth, J., Ottinger, M., and Mack, B.: The potential of open geodata for automated large-scale land use and land cover classification, Remote Sens., 11, 2249, 2019.

Mack, B., Leinenkugel, P., Kuenzer, C., and Dech, S.: A semiautomated approach for the generation of a new land use and land cover product for Germany based on Landsat time-series and Lucas in-situ data, Remote Sens. Lett., 8, 244-253, 2017.

Meroni, M., d'Andrimont, R., Vrieling, A., Fasbender, D., Lemoine, G., Rembold, F., Seguini, L., and Verhegghen, A.: Comparing land surface phenology of major European crops as derived from SAR and multispectral data of Sentinel-1 and-2, Remote Sens. Environ., 253, 112232 , https://doi.org/10.1016/j.rse.2020.112232, 2021.

Orgiazzi, A., Ballabio, C., Panagos, P., Jones, A., and FernándezUgalde, O.: LUCAS Soil, the largest expandable soil dataset for Europe: a review, Eur. J. Soil Sci., 69, 140-153, 2018.

Pflugmacher, D., Rabe, A., Peters, M., and Hostert, P.: Mapping pan-European land cover using Landsat spectral-temporal metrics and the European LUCAS survey, Remote Sens. Environ., 221, 583-595, 2019.

Scarnò, M., Ballin, M., Barcaroli, G., and Masselli, M.: Redesign sample for Land Use/Cover Area frame Survey (LUCAS) 2018, in: Statistical Working Papers, Publications Office of the European Union, Luxembourg, https://doi.org/10.2785/132365, 2018

Weigand, M., Staab, J., Wurm, M., and Taubenböck, H.: Spatial and semantic effects of LUCAS samples on fully automated land use/land cover classification in high-resolution Sentinel-2 data, Int. J. Appl. Earth Obs. Geoinf., 88, 102065, https://doi.org/10.1016/j.jag.2020.102065, 2020.

Zillmann, E., Gonzalez, A., Herrero, E. J. M., van Wolvelaer, J., Esch, T., Keil, M., Weichelt, H., and Garzón, A. M.: PanEuropean grassland mapping using seasonal statistics from multisensor image time series, IEEE J. Sel. Top. Appl. Earth. Obs. Remote Sens., 7, 3461-3472, 2014. 